(6) OPEN ACCESS

\title{
Doubly distributing special obligations: what professional practice can learn from parenting
}

\author{
Jon Tilburt, ${ }^{1}$ Baruch Brody ${ }^{2}$
}

Mayo Clinic, Rochester, Minnesota, USA 2Department of Philosophy, Rice University, Houston, Texas, USA

\section{Correspondence to} Dr Jon Tilburt, Mayo Clinic, 200 First Street SW, Rochester, MN 55905, USA;

tilburt.jon@mayo.edu

Received 18 August 2015 Revised 3 March 2016 Accepted 7 April 2016 Published Online First 28 April 2016
Check for updates

To cite: Tilburt Brody B. J Med Ethics 2018:44:212-216.

\begin{abstract}
A traditional ethic of medicine asserts that physicians have special obligations to individual patients with whom they have a clinical relationship. Contemporary trends in US healthcare financing like bundled payments seem to threaten traditional conceptions of special obligations of individual physicians to individual patients because their population-based focus sets a tone that seems to emphasise responsibilities for groups of patients by groups of physicians in an organisation. Prior to undertaking a cogent debate about the fate and normative weight of special obligations and a traditional ethic for contemporary healthcare, we need a deeper examination of what the traditional ethic of special obligations really means. Here we offer a conception of 'doubly distributed' special obligations. Physicians and similarly minded healing professionals abiding by a traditional ethic have always spread their devotion and attention across multiple patients and have shared responsibilities with physician and non-physician colleagues in much the same way devoted parents have frequently distributed their special obligations across multiple children and across multiple parents. By taking up the extended analogy of parent we argue that doubly distributing special obligations need not contradict the possibility of special obligations in restructured collective forms of healthcare delivery and financing.
\end{abstract}

A traditional ethic of medicine is based on the notion of physicians having special obligations to serve the best interests of individual patients independent of societal or institutional pressures.

Contemporary trends in US healthcare financing and reform could shift the emphasis of physician obligation away from individuals in a way that threatens altogether the viability of special obligations to individuals inherent in a traditional ethic of medicine.

The tensions between incentives for group performance and obligations to individuals have been institutionalised in national policy objectives. For instance, the Institute of Healthcare Improvement's 'Triple Aim'(ie, patient experience, population health and reduced cost) - if applied to individual physician obligations-implies a responsible physician might try to toggle back and forth between serving individual patients and serving a population, doing one's best to reconcile the needs and interests of both simultaneously. This possibility was raised in the 1990s in the era of managed care and health maintenance organisations. ${ }^{1}$ Similar potential exists in accountable care organisations (ACOs). Physician employees of ACOs would serve populations with a backdrop of incentives that favour groups over individuals.
The medical literature has tried to grapple with this ethical reality, ${ }^{1-7}$ identifying the tensions in modern healthcare financing. ${ }^{8-11}$ Typically, commentators reiterate the primacy of patient welfare, leaving the tension with other organisational obligations unaddressed. Special obligations to individuals and the edifice of a traditional ethic of medicine it supports may just be obsolete for $21 \mathrm{st}$ century medicine.

We posit that both supporters and detractors of a traditional ethic presuppose an inaccurate stereotype of what a traditional ethic of medicine actually has required and will require of physicians. That stereotype-special obligations require a highly demanding, self-effacing, individually focused devotion-mischaracterises an implied, sanctioned, nuanced and long-standing functional consensus that was always present in the traditional ethic that is much more nuanced. When unpacked the unsaid assumptions of what the traditional ethic has always implied about special obligations provides plenty of space for distributing special obligations across groups of patients and across teams of clinicians. Therefore, challenges to special obligations posed in recent health policy reforms can be met with a more transparent, robust and nuanced construal of special obligations always implied in but never spelled out in a traditional ethic of medicine. We present an analogy from the special obligations of parents to illustrate our case.

\section{DOUBLY DISTRIBUTED SPECIAL OBLIGATIONS}

A traditional ethic of medicine assumes physicians have special obligations to serve the best interests of their individual patients. These obligations apply to the particular social role of physician. Special obligations are foundational and partly constitutive of what it means to be a physician professional.

Despite the importance of special obligations in the self-definition of the medical profession, the ways special obligations get talked about (whether using the term or not) seem to imply they are singular and near absolute. The singularity of special obligations is illustrated in this quote from an early 20th century speech by William J Mayo: "the best interest of the patient, is the only interest to be considered." ${ }^{12}$ Peabody's famed lines about the care of 'The Patient' ${ }^{13}$ highlight the singularity implied in special obligations that carries through to Pellegrino and Thomasma: "The physician must always place the good of the patient over other goods" (emphasis added). ${ }^{14}$

Rhetoric surrounding the traditional ethic of medicine has nearly always implied special obligations are near absolute in gravity and scope. If my patient needs me, I am there. Full stop. 
Near absolute style assumptions about special obligations fit common lingering stereotypes about physicians. We conjure images of physicians who never sleep, take vacations, have families or learn hobbies. They are required to put their life at risk with reckless abandon for the sake of patient protection. They are absorbed by their professional calling. So reads the rhetoric around a traditional ethic. Then the popular consciousness quickly leaps to the absolute normative conclusion: a physician's interest or obligation to her individual patient trumps other legitimate interests and obligations. Cast in this way, a traditional ethic of medicine aligns with an ethos and mentality of a solo practice physician addressing urgent, acute, serious illnesses in a remote location circa 1910. Although we do not really believe it is true, our language surrounding traditional ethic still uses those stereotypical assumptions.

But the singular and near-absolute conception of how special obligations have been cast was never actually expected of physicians. 'Doing what is best for the individual patient' was a generalisation subject to all sorts of exceptions. ${ }^{15}$ Physicians are paged while taking care of other patients. Surgeries on prepped patients are postponed when more urgent cases present. Anaesthesiologists may even disregard the pain relief of some low-risk women in labour to care for women in labour requiring emergency surgery. Scarcity and triage show us that obligations of individual physicians are neither singular nor absolute. These limitations do not, per se, constitute a fundamental threat to the idea of special obligations. They do suggest that the service and self-effacement in medicine's special obligations and articulated in a traditional ethic has limits and may be distributed across patients despite the ways we may talk about them or imagine them in the popular consciousness.

Special obligations are distributed in another sense tooacross physicians and other members of the healthcare team. Doctors 'cover' for each other; they 'hand off' their special obligations to another or transfer care to another. Sometimes patients are assigned to a team. In complex care, multiple specialists share responsibility for coordinating a patient's evaluation and management. Sometimes a physician is obliged to relinquish entirely his control in the patient's care. Analogously, physicians have other legitimate and exemplary obligations in life outside of their clinical work-teaching trainees, important family relationships, institutional roles, public health advocacy, or even lecturing in ethics-that necessitate delimiting their availability to patients. In each of these cases, the special obligation to the individual patient is distributed among (or transferred across) a care team. Special obligations can be distributed not just across patients but also across professionals.

This doubly distributed model of special obligations-spreading obligations across patients and across teams-gives a more realistic account of what a traditional ethic has meant and surely now means in contemporary care. Contemporary restructuring of medical practice will require that the distributed features of special obligations be examined and applied with attention and rigour.

\section{THINKING CLEARLY ABOUT SPECIAL OBLIGATIONS: THE PARENTAL ANALOGY}

To illustrate the doubly distributed nature of physician special obligations, consider parenting. Intimate relationships have been used commonly to analyse obligations. Several generalisations about parents' roles establish it as a suitable although imperfect analogue. Both relationships-parenting and patient care-involve social roles that we think are important and praiseworthy. Both involve asymmetry of power. Both require self-effacement. We take up this analogy acknowledging this as an exercise in descriptive ethics-describing gross social norm generalisations from one sphere of life as a means of thinking clearly about the social norms in another sphere of life even if the arguments for the specifics of those norms are not airtight. For reasons of brevity we invite the reader to go along with these generalisations for the purposes of deepening our understanding of special obligations in healthcare.

Below we describe five commonly accepted generalisations about parental special obligations to deepen our appreciation of the doubly distributed nature of physicians' special obligations.

\section{Special obligations of parents are legitimately limited}

In the simplest scenario, an individual parent holds special obligations to an individual child. A parent is charged with protecting and nurturing the child's well-being as well as with fostering the child's growth. The parent's devotion, commitment and attention are selective and weighted heavily to her child, not all children.

Yet, a parent's special obligations are not limitless. Parents slow down, delay and forego attention to many of their own needs and desires in the fulfilment of their special obligations to their children. But parents have a right to not neglect indefinitely their own emotional, physical and developmental needs. There are times when a parent choosing to spend time and money on her own career development and workforce re-entry instead of, for instance, investing more time, energy and money into an advanced, more intensive music training programme for a child could be praiseworthy. Parents also have other legitimate obligations to professional colleagues, the community, or one's own parents that may limit devotion to a child's needs.

Children can benefit when their parents exercise other obligations at the expense of the child's non-urgent concerns. A child learns he is one node in his parent's matrix of moral relationships—a close and especially connected node-but just one.

A parent's limited time, devotion and energy must account for the strong and weighty obligations to her child for sure, but at least the time and energy are limited and thus require discernment to meet a child's needs. Of course the opposite is true too. Too limited time, attention and devotion can hurt children and can be cause for us to ascribe blame to parents for neglecting them. If a parent's attention is diverted from his child for frivolous or destructive behaviour, he could be blamed for not fulfilling his special obligations. But parents having other needs and obligations outside of a parenting role per se need not undermine the existence of fundamental special parenting obligations.

\section{Needs are different than wants and other goods}

Special obligations of parents pertain to taking responsibility for meeting the basic needs essential for a child's development. ${ }^{i}$ Parents may need to sacrifice a considerable amount of time, finances and energy to ensure true needs are met. How that plays out may vary from culture to culture, but many basic features persist across cultures.

What a child wants and what is good for a child are different, subtler matters. If a child does not get their favourite meal

\footnotetext{
${ }^{\mathrm{i}}$ We cannot give an exhaustive account of what is and is not covered in 'basic needs', but merely assert that a distinction of what a child needs, wants and is good for her is central to thinking about parental obligations. We believe that such a distinction is best based on a Sen/ Nussbaum approach. ${ }^{16}$
} 
tonight, they are not harmed, and parents are not negligent, so long as the child's basic biological, economic, emotional and spiritual needs are being attended to. Similarly, it might be good for a child to have their teeth straightened; that is neither a need nor a want per se, but it is still good. Moreover, the longterm developmental interests of children are better served when parents refrain from satisfying all 'wants' of a child. Doing so can create space for that child to develop his own capacity to fulfil the want independently.

Parents may not be required to give up as much for a child's wants compared with a true need. However, a child's wants are important and should not be ignored. Part of good parenting involves providing opportunities for that child to flourish through exploring and fostering preferences, aptitudes and interests. Thus, a parent must make a lot of judgement calls about the merits of a 'want' in deciding whether or how to satisfy it. A child who delights in making things with his hands, but never is allowed to explore crafts or Legos may not flourish. Other wants may not merit any attention or resources at all. Thus, great parenting is a dance that incorporates wants, but does not necessarily cater to them at every moment.

Moreover, distinguishing needs from wants or other goods in particular circumstances may not be easy. For instance, one of us has a 12-year-old child who at the age of 10 years declared herself a vegetarian. By our lights, parents are not strictly obliged to provide a perfect vegetarian menu for their children who choose to be vegetarian. However, systematically withholding viable plant-based protein sources when financial resources are not a constraint probably disrespects who that child sees herself to be. Respecting that child's considered and sustained preference while strictly not a 'need' represents more than a spurious 'want' and seems to take on some features of need, want and good.

\section{Sufficient satisfaction of special obligations is a function of resources and context}

Appropriate exercise of parental special obligations is also a function of the resources, culture and context. In a particular country routine secondary education may not be a basic need for children. In other settings, it might be. Likewise, what constitutes an acceptable risk-good hygiene, enough oversight and 'adequate' provision of care-are embedded in social expectations and conditions of one's social context at a given point in time. Behaviours that might be grounds for a parental neglect claim in North America in 2015 may look quite different in the developing world (or North American in 1950), and rightly so.

\section{When others of one's own children are involved, parents must factor in their special obligations to each of the individual children}

The obligations of a parent often involve multiple children. When children join a family, a parents' capacity to love may grow, but their capacity to exercise their special obligations to each of the individual children in a timely manner may be limited by competing obligations. Urgency of needs, prospect of benefit, commitment to fairness and existence of alternative options for meeting a need may influence how parents discharge their special obligations. In addition, competing legitimate nonparental role obligations may influence how parents discharge their special obligations. If a diaper needs to be changed for Suzie, snack can wait for Charlie. How much a child stands to benefit from a parent's time and attention in a given instance bears consideration as well. And asking prudential questions like 'will the satisfying of this need require a lot or a little parental energy?' is important too. Similarly, if a child has a need, but that parent does not have the resources to address the need, the parent may need to 'triage' and focus on those who can be benefited at a given moment. Wise parents will assess how their attention is spread across children over time, asking "am I giving each child their due attention and devotion suitable to their developmental stage and in a way that avoids perception of favoritism?" Parents may also delegate care to another parent or family member to meet their full range of obligations to children and others. Even if the specific parenting maxims suggested here are not shared by all parents, they are reasonable maxims and, for the sake of argument, establish the plausibility of distributed special obligations in parenting.

In non-urgent situations where no basic needs are at stake, great parenting will require allowing kids to experience and grow from their strengths through the undivided attention of the parent, which may require a sibling to wait in the fulfilment of their own wants. That attention may require periods of focused energy on a particular child that make equity of time and attention difficult to achieve.

\section{When multiple parents are involved other factors come into play}

When multiple parents are involved, decisions of how to apportion time, energy and attention between the parents are negotiated based on parental and child wishes, legitimate competing parental demands, child developmental phase, parental life goals/trajectory/fulfilment, household income earning considerations, as well as the skills/aptitudes of respective parents, and desire for continuity. Such determinations are dynamictogether discerning what is best to meet the needs of children at various developmental phases. At one phase in a child's life, one parent in a two-parent relationship may spend a larger amount of time with that child. Sometimes parents negotiate to have the 'less competent' parent assume responsibility for the children so the 'more competent' parent can have a break or pursue other noble goals as discussed above (even if the children would prefer the more competent caregiver). A child's 'want' for one parent must be considered in light of all the factors discussed. Distributing and redistributing the time and attention entailed by special obligations across parents is normal and healthy for caregivers and those being cared for. Such sharing can be done with integrity that does not compromise a parent's special obligations.

Some might question our analogy based on the incongruences between clinical and familial relationships. Admittedly much more would need to be worked through to determine at what point the analogy breaks down. Nonetheless the analogy of parenting can be instructive to help in thinking about distribution of physician special obligations.

\section{DOUBLY DISTRIBUTED SPECIAL OBLIGATIONS: IMPLICATIONS FOR MEDICINE}

If the lessons of parental obligations are a guide, we can begin to see how the special obligations of physicians and other healthcare professionals are influenced by, but need not be obliterated by, changing structures of healthcare where groups of physicians are responsible for (sometimes large) groups of patients. In reality, the coming mandate for bundled payment such as ACOs' is not dissimilar to how physician special obligations have played out in group practice models for decades. Below we illustrate how special obligations might play out for medicine using a doubly distributed conception. 


\section{Special obligations of physicians are legitimately limited}

To fulfil their special obligations to patients, physicians need not have their patient care obligations be all encompassing. In fact, physicians might be better physicians if their career obligations were not all encompassing. Physicians with no avocational passions- who never, fish, run, read, play-may exhibit less than fully human (or humane) attributes in their work. It is good that physicians have interests and goals outside of their patient care role. It is right that they attend to institutional, civic, familial and collegial obligations beyond direct patient care. While this may seem obvious, the articulation of this reality in the traditional ethic in the 20th century was largely absent.

\section{Needs are different than wants}

We might also note that just as for children, distinguishing patient wants from patient needs may be crucial to discharging a physician's special obligations. Patients may want things that are less beneficial or are even harmful, or they may feel entitled to technology or services that they are not entitled to. Physicians who help patients live within limits that are in their own best interests are taking their special obligations seriously. Deprivation of wants does not violate special obligations per se. Nevertheless, patient preferences are important. This problem is particularly difficult when the patient needs to be treated for a problem but wants for various reasons a suboptimal treatment. We do not deny that how and under what circumstances to accommodate a patient preference for suboptimal treatment may be a difficult problem. The same basic struggles and complexity hold in parenting. Ought a parent to allow a 14-year-old to waste $\$ 200$ of his hard-earned money on a seemingly frivolous purchase? When patients want what appears, from a medical perspective, to be suboptimal care, when can/should a physician accommodate that?

Several factors may weigh into a parent's or clinician's determination of whether to accommodate such a preference: Risk of irreversible harm (both magnitude and probability), coherence with previously stated wishes and the robustness of the value set on which the preference is based (eg, Does the patient's whole worldview line up with this preference such that not allowing it would be an affront to his/her way of life?) seem like reasonable candidates.

We also acknowledge it is difficult to always and consistently draw the needs versus wants distinction clearly both in parenting and in practice because in reality children and patients experience wants as needs. For this and other reasons, making the application of this distinction may seem sometimes arbitrary. If a want is so closely aligned with how the patient or the child sees himself or herself while the physician/parent still regards it as a want, friction, misunderstanding or conflict could ensue. That reality holds equally for both parenting and medicine. The fact that the distinction may seem arbitrary in some particular circumstance does not negate the legitimacy and relevance of the general intuition.

Navigating the wants versus the needs of patients can be challenging. Part of being a professional involves doing what is right for the patient regardless of the incentives. A patient may 'want' arthroscopy for her arthritis, but that does not necessarily mean she needs it, since the data supporting it are questionable. ${ }^{17}$ However, ignoring uncontrolled arthritis pain and the patient's desire to do something about it would not be right either.

Sometimes a patient's need may be met by not using the medical system to achieve that need. The temptation to commit the equivalent of 'hover parenting' in medicine and thereby make a medical issue out of every aspect of a patient's life, wellness and self-care is a real challenge. Discharging special obligations may at times mean not intervening and instead cultivating the capacity of the patient to meet their own needs.

\section{Sufficient satisfaction of special obligations is a function of resources and context}

What constitutes the basic needs of a patient is a function of the resources and context. Basic postmyocardial infarction care may look quite different in Houston than it does in Havana. Thus, what it means to be an attentive caregiver is a function of the circumstances of caring. Physicians can no more escape this than parents can. Systems, insurance and documentation all play into how care responsibilities of the physician are met.

\section{When others of one's own patients are involved, physicians must factor in their special obligations to each individual patient}

When a physician takes on multiple care relationships, each with its special obligations, the time and attention required to discharge those obligations will need to be distributed across individuals. Acuity of need, commitment to fairness, likelihood of benefit and ability to fulfil the service, all factor into how time and attention are apportioned with patients. This fact may weaken but does not obliterate the idea of special obligations. For generations physicians have managed these limitations implicitly, and doing so wisely is not easy, but can be done. For most patients, most of the time, this distribution is manifest by having to wait to see the doctor. Doctors are often late, sometimes to the point of disrespecting scheduled patients. That lateness is in part justifiable to the extent that the delays were not foreseeable and the other patients delaying the physician were deserving of and benefiting from that attention. These realities imply a real distributional constraint on how physicians meet their individual obligations to members of a group that did not begin with bundle payments or population health nor do they spell the end of special obligations.

\section{When physicians practise in groups, responsibilities can be shared and negotiated}

Thankfully, medicine is not practised alone. The time, care and attention needed to meet a physician's special obligations can and often must be distributed across different responsible caregivers. Different clinicians on a team can share responsibility for a patient or multiple patients. Those roles are not always intuitive. For sharing to be constructive, roles need to be negotiated. Contractual obligations, schedules, expertise, patient comfort level, degree of prior bond with the patient may all factor into how special obligations are operationalised across team members. Who 'owns' the patient's care can vary over time. When should the patient with chronic respiratory disease be transferred back to the medicine service after elective knee replacement? These are not easy questions that must be negotiated among colleagues, but all speak to the implied reality that operationalising special obligations spreads time and attention for patients across multiple caregivers.

In these circumstances patient preferences are important but not absolute. A patient may prefer one cardiologist to another, but schedules may make seeing the preferred cardiologist more difficult. While systems should not dictate whom the patient sees, they should account for patient preference and promote continuity, especially in non-urgent situations. Having the continuity of a relationship to rapidly incorporate patient 
preferences is crucial to humanising systems that distribute care responsibilities.

While there may be a risk of physicians who practise in ACOs misreading the performance incentives and reflexively medicating an individual patient against her wishes to 'help his numbers', those same bundled payment plans could provide a more patient-centred, holistic team approach to empowering the patient's daily life. Arguably, rather than medicating marginally elevated blood pressure to try to achieve a short-term performance target, systems and teams that support chronic disease selfmanagement (as many ACOs do) could offer programmes that promote diet and lifestyle counselling first that over time could increase self-care capacity and thereby effect a much greater impact on a patient's long-term health outcomes. Conversely, if Shared Savings Programmes turn out to create their own perverse incentives for physicians to overtreat or disrespect individual patients, physicians would have an obligation to resist those incentives and instead advocate for necessary changes in ACO evaluation metrics, even if it meant not meeting performance targets. Such advocacy would rightly derive from and would find its support in the enduring special obligations physicians feel is a part of their job even in imperfect systems.

There will be challenges in upholding physician special obligations in the midst of incredible system change. Is it possible to salvage what was good from the individual model of care to cultivate a sense of 'taking ownership' for a patient's management when so many aspects of a patient's care are spread across so many team members? Can we still image medicine as a profession of self-sacrifice when physicians increasingly feel less control and less emotional investment in the future of their practice? Will physicians be able to believe and convey a sense of the unique needs of each patient when they are encouraged to standardise care and manage populations? Will the benefits of relationship continuity succumb to the burgeoning bureaucratic need for 'throughput'? These questions are not trivial and speak of the hard challenges ahead for the profession. These and other legitimate challenges can be faced with a realistic and robust commitment to special obligations of healthcare professionals as our doubly distributed account outlines.

\section{CONCLUSION}

Groups of doctors caring for groups of individuals appears to be the trend for the foreseeable future of US healthcare. Contemporary trends in US healthcare financing and reform may shift physicians' focus away from the needs of individuals to populations. When physician special obligations are distributed across multiple patients and multiple caregivers, those obligations are stretched, not dissolved. Those shifts will need to be navigated. ${ }^{18}$ Those shifts may have favourable economic effects and bring with them disconcerting relational effects. Adding relationships need not dissolve existing special bonds and indeed can foster greater resolve to care in a particular and heartfelt way in much the same ways that happen when families grow. By thinking clearly and imaginatively about the special obligations of physicians to patients, the profession can navigate the very real tensions, disagreements and upheaval bearing down upon it with devotion to a traditional ethic of medicine realistically interpreted within the realities of 21 st century systems.

Contributors JT and BB have both contributed to this paper, and are solely and equally responsible for the content of the paper.

Funding Greenwall Foundation (Faculty Scholars programme).

Competing interests None declared.

Provenance and peer review Not commissioned; externally peer reviewed.

Open Access This is an Open Access article distributed in accordance with the Creative Commons Attribution Non Commercial (CC BY-NC 4.0) license, which permits others to distribute, remix, adapt, build upon this work non-commercially, and license their derivative works on different terms, provided the original work is properly cited and the use is non-commercial. See: http://creativecommons.org/ licenses/by-nc/4.0/

\section{REFERENCES}

1 Povar GJ, Blumen H, Daniel J, et al. Ethics in practice: managed care and the changing health care environment: medicine as a profession managed care ethics working group statement. Ann Intern Med 2004;141:131-6.

2 Sulmasy DP. Physicians, cost control, and ethics. Ann Intern Med 1992;116: 920-6.

3 Daniels N, Sabin J. The ethics of accountability in managed care reform. Health Affairs 1998;17:50-64.

4 Hall MA, Berenson RA. Ethical practice in managed care: a dose of realism. Ann Intern Med 1998;128:395-402.

5 Emanuel EJ, Brett AS. Managed competition and the patient-physician relationship. N Engl J Med 1993;329:879-82.

6 Emanuel EJ, Dubler NN. Preserving the physician-patient relationship in the era of managed care. JAMA 1995;273:323-9.

7 Clancy CM, Brody H. Managed care. Jekyll or Hyde? JAMA 1995;273:338-9.

8 Glasson J, Plows CW, Clarke OW, et al. Ethical issues in managed care: council on ethical and judicial affairs, American medical association. JAMA 1995;273:330-5.

9 Snyder L. American college of physicians ethics manual: sixth edition. Ann Intern Med 2012;156Pt 2):73-104.

10 Charles SC, Lazarus JA. Reframing the professional ethic: the Council of Medical Specialty Societies consensus statement on the ethic of medicine. West J Med 2000;173:198-201.

11 AMA Council on Ethical and Judicial Affairs. Opinion 8.131 Professionalism in Health Care Systems, 2014 (14 July 2015).http://www.ama-assn.org/ama/pub/ physician-resources/medical-ethics/code-medical-ethics/opinion8131.page?\#

12 Saint Marys Hospital. Collected papers by the staff of Saint Mary's Hospital, Mayo Clinic: W.B. Saunders Company, 1911.

13 Peabody FW. The care of the patient. JAMA 1927:88:877-82.

14 Pellegrino ED, Thomasma DC. For the patient's good: the restoration of beneficence in health care. New York: Oxford University Press, 1988.

15 Wendler D. Are physicians obligated always to act in the patient's best interests? J Med Ethics 2010;36:66-70.

16 Nussbaum M. Capabilities as fundamental entitlements: sen and social justice. Feminist Econ 2003;9:33-59.

17 Kirkley A, Birmingham TB, Litchfield RB, et al. A randomized trial of arthroscopic surgery for osteoarthritis of the knee. N Engl J Med 2008;359:1097-107.

18 DeCamp M, Farber NJ, Torke AM, et al. Ethical challenges for accountable care organizations: a structured review. J Gen Intern Med 2014;29:1392-9. 